\title{
Massive star formation with Spitzer/GLIMPSE
}

\section{Rémy Indebetouw ${ }^{1}$, Barbara A. Whitney ${ }^{2}$, Thomas P. Robitaille ${ }^{3}$, Ed Churchwell ${ }^{4}$ and the GLIMPSE team}

\author{
${ }^{1}$ Astronomy Department, University of Virginia, PO Box 318, Charlottesville, VA, 22903, USA \\ email: remy@virginia.edu \\ ${ }^{2}$ Space Science Institute, 4750 Walnut St., Suite 205, Boulder, CO 80301 \\ email: bwhitney@spacescience.org \\ ${ }^{3}$ School of Physics and Astronomy, University of St Andrews, North Haugh, KY16 9SS, St \\ Andrews, United Kingdom \\ email: tr9@st-andrews.ac.uk \\ ${ }^{4}$ Astronomy Department, University of Wisconsin, 475 N Charter Street, Madison, WI 53706 \\ email: ebc@astro.wisc.edu
}

\begin{abstract}
The Spitzer Space Telescope is very efficient at moderate-depth infrared mapping. The Galactic Legacy Infrared Midplane Survey Extraordinaire (GLIMPSE) and other projects have now imaged most of the massive star formation regions in the inner Galaxy. In this case, quantity is quality - the large datasets allow statistical and global analysis of star forming regions that was previously difficult. Data such as the GLIMPSE survey is proving useful to study different evolutionary stages of massive star formation, from pre-protostellar clouds through feedback and triggered star formation. We discuss selected team results relevant to each of these stages: mapping infrared dark clouds and the mid-IR extinction law, studies using radiative transfer models of the entire protostellar population of star forming regions such as M16 and M17 (see also Whitney, this conference), feedback and star formation in the G305 and RCW79 giant HII regions, and GLIMPSE's comment on even older objects such as debris disks and PN. Continuing similar analysis will hopefully lead to better understanding of large-scale questions of Galactic star formation such as the total star formation rate and the relationships between star formation and Galactic structure.
\end{abstract}

Keywords. Galaxy: general, stars: formation, infrared: stars, HII regions

\section{Introduction}

Massive stars form in complex environments (nearly always protoclusters). Space densities of stars in Galactic clusters and of prestellar clumps in star forming regions (Motte et al. 1998 and Motte, this volume) suggest that interactions between protostellar objects are important in massive star formation (see contributions in this volume including Bonnell and Dobbs). The radiative and mechanical feedback from massive protostars almost certainly affects the formation of other objects in the protocluster (the most commonly known example being the Orion proplyds), if not their own formation. These considerations encourage, if not necessitate, the global and statistical analysis of (massive) star forming regions: one must study the simultaneous evolution of the multiple protostellar and young stellar objects of different mass. Such global study requires fast (wide-field) sensitive imaging.

The rapid timescales of massive star formation necessitate long-wavelength observations. Accretion rates are one to three orders of magnitude larger than in solar-mass stars 
(Maeder \& Behrend 2002,Tan \& McKee 2004), and the very short Kelvin-Helmholtz contraction time suggests that at least the central part of an accreting massive protostellar object will begin nuclear burning within a few $10^{5}$ years. Thus all the "action" occurs before the surrounding cloud has had time to be dispersed, at typical extinctions $\mathrm{A}_{V} \gtrsim 30$.

The Galactic Legacy Infrared MidPlane Survey Extraordinaire (GLIMPSE) illustrates the power of the Spitzer Space Telescope to perform the wide-field long-wavelength $(\gtrsim 2 \mu \mathrm{m})$ photometry required to understand massive star formation across our Galaxy. GLIMPSE is one of Legacy programs associated with Spitzer - these large programs were funded early and with no proprietary period to the data, to facilitate widespread community access to enhanced data products. The GLIMPSE survey (PI Ed Churchwell, University of Wisconsin, see Benjamin et al. 2003 for a description of the project) surveyed the inner Galaxy (10 deg $<|l|<65 \mathrm{deg},|b|<1 \mathrm{deg})$ at 3.6, 4.5, 5.8, and $7.9 \mu \mathrm{m}$ at $\sim 2^{\prime \prime}$ resolution using IRAC (Fazio et al. 2004). The GLIMPSE team produced a point source Catalog (high-reliability) and Archive (more complete but still very reliable). The latter reaches approximate brightness limits of 14.2, 14.1, 11.9, and 9.5 mag (0.6, 0.6, 2.0, and $10 . \mathrm{mJy}$ ) at $3.6,4.5,5.7$, and $7.8 \mu \mathrm{m}$, largely due to confusion of unresolved point sources in the two shorter-wavelength bands, and due to background and sensitivity in the two longer-wavelength bands. This resolution and sensitivity enables one to study the stellar content of a typical massive star-forming region $\left(10^{5} \star / p c^{3}\right)$ out to $2.5 \mathrm{kpc}$ down to 2-3 solar-mass YSOs. Note that this particular figure is limited by spatial resolution, not sensitivity: GLIMPSE can study solar-mass YSOs that are isolated or in loose groups (less than $500 \star / p c^{3}$ ) out to $7 \mathrm{kpc}$, and the most massive (OB) members of star forming regions can be studied all the way across the Galactic disk ( $\gtrsim 15 \mathrm{kpc})$.

\section{Tools to exploit GLIMPSE data}

\subsection{A study of interstellar extinction}

Efficient use of a survey such as GLIMPSE requires specialized tools. In the specific case of observations in the $3-10 \mu \mathrm{m}$ regime, the extincting and reddening effects of dust needed to be better understood before the spectral energy distributions of reddened objects could be interpreted. We examined the wavelength dependence of interstellar extinction towards several sightlines dominated by the diffuse ISM in Indebetouw et al. (2005). The method used exploited the large number of point sources with photometry between 1.2 and $8.0 \mu \mathrm{m}$ in the combination of GLIMPSE and the 2MASS surveyt, and would not have been possible without wide-field photometric imaging. Differential reddening to point sources along a (several square arcminute) sightline spread those point sources out along a line in color-color space. The slope of that line in a plot of $[\lambda]-K$ vs. $J-K$ is the relative color excess $\frac{E_{[\lambda]-K}}{E_{J-K}}$. The color excesses are related to the wavelength dependent extinction $A_{[\lambda]}$ by

$$
\frac{A_{[\lambda]}}{A_{K}}=\left(\frac{A_{J}}{A_{K}}-1\right) \frac{E_{[\lambda]-K}}{E_{J-K}}+1 .
$$

Various observational studies and dust theories find a range of $A_{J} / A_{K}$ between 2.25 and 2.75 (Cardelli et al. 1989, Fitzpatrick 1999, Draine 2003). We determine the ratio $A_{J} / A_{K}$ directly from our data by fitting the locus of red clump giants in a color-magnitude diagram.

$\dagger$ This publication makes use of data products from the Two Micron All Sky Survey, which is a joint project of the University of Massachusetts and IPAC/Caltech, funded by NASA and the NSF 

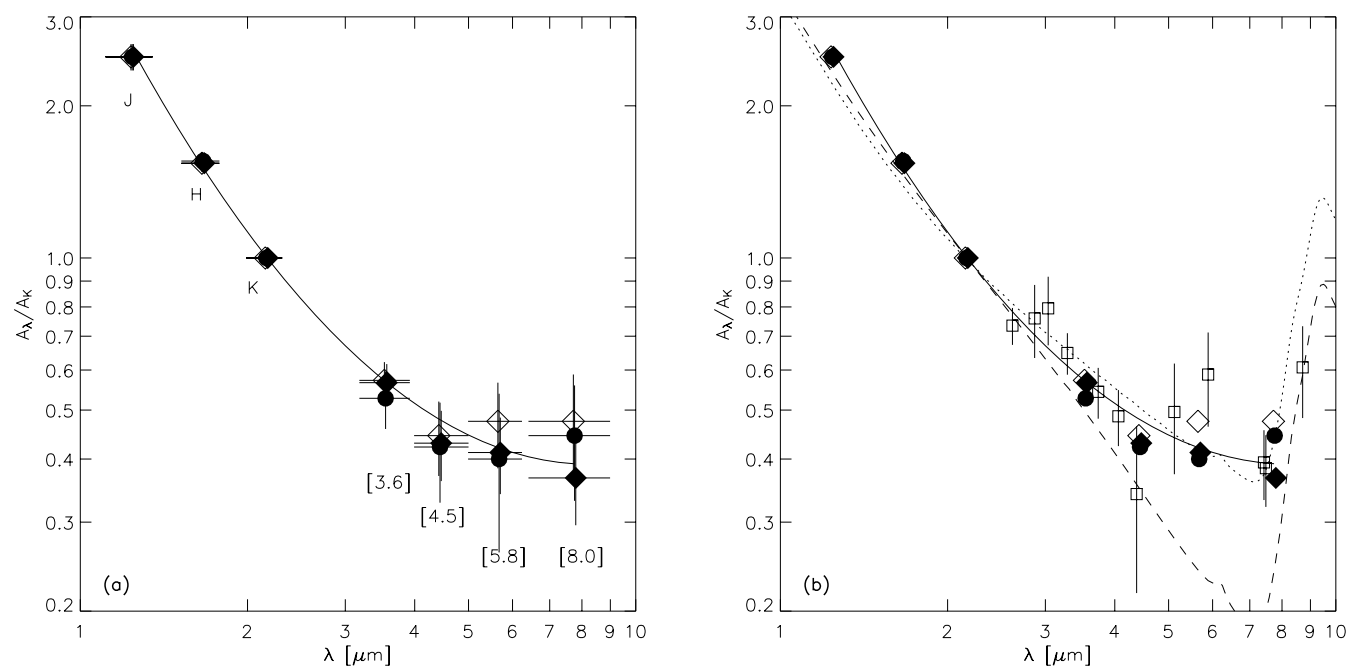

Figure 1. In the first panel, symbols show the derived relative extinction values for RCW 49 (solid circles), the field near $l \sim 284 \mathrm{deg}$ (solid diamonds), and the field near $l \sim 42 \mathrm{deg}$ (open diamonds). The open and solid diamonds coincide at $\lambda=3.5 \mu \mathrm{m}$. The lines show fits to the mean field (solid) and RCW 49 (dot-dashed). Horizontal bars indicate the filter widths and the fact that we have only sampled the wavelength dependence of interstellar extinction at those discrete filters. In the second panel, the measurements of Lutz et al. (1996) have been added (squares with error bars), as well as theoretical curves from Weingartner \& Draine (2001) for $R_{V}=5.5$ (their case "A" dashed, their case "B" dotted).

Figure 1 shows the derived extinction curve for the field stars in three directions. The uncertainties are a combination of uncertainty in determining $A_{J} / A_{K}$, variation in fit results depending on the exact part of the sky, stellar population, and fit algorithm, calculated uncertainty of the fit coefficients, and errors in GLIMPSE and 2MASS photometry (in order of decreasing significance). There is good agreement between the three determinations of $A_{[\lambda]} / A_{K}$, in two very different directions of the Galactic plane, and even towards a giant HII region.

The main feature of our derived extinction is that there is greater relative extinction at $\sim 5 \mu \mathrm{m}$ than a power-law extending from the near-IR would indicate. This has been observed towards the Galactic center (Lutz et al. 1996), towards an inner Galaxy field by ISOGAL (Jiang et al. 2003), and ongoing studies with Spitzer/IRAC indicate a similar and perhaps even stronger effect in molecular clouds (Megeath and Huard 2005, private communications).

\subsection{Model Grid}

The most important tools required to exploit survey data are automated processors to analyze many sources at once. The GLIMPSE data processing pipeline is the zeroeth order part of this machinery, and was developed over several years by the core team of Marilyn Meade, Brian Babler, Barbara Whitney, Christer Watson, Mark Wolfire, Mike Wolff, and Rémy Indebetouw. The next step is to identify all of the point sources extracted by the pipeline as extincted stellar photospheres, young stellar objects of some evolutionary state, or potentially something else. This is accomplished by comparing each GLIMPSE point source with a large grid of synthetic spectral energy distributions (SEDs) of (mostly) young stellar objects - see Whitney, this volume for dicussion. 
Table 1. Range of parameters covered by protostellar model grid

\begin{tabular}{lc}
\hline stellar mass* $^{*}$ & $0.1-40 \mathrm{M}_{\odot}$ \\
stellar age* & $10^{4}-10^{7}$ years \\
envelope infall rate & $10^{-4}-10^{-9} \mathrm{yr}^{-1 *} \mathrm{M}_{\star}$ \\
disk mass & $10^{-1}-10^{-8} \mathrm{M}_{\star}$ \\
disk radius & $10-1000 \mathrm{AU}$ \\
bipolar cavity opening angle & $10-50$ degrees \\
inclination angles & 10 \\
\hline
\end{tabular}

* using prestellar evolutionary tracks of Siess, Dufour, \& Forestini(2000)

Spectral energy distributions (SEDs) of young stellar objects are produced using the Monte-Carlo radiative transfer code described in Whitney at al. (2003a) (see also Whitney et al. 2003b, Whitney et al. 2004 and Whitney, this volume). The model solves for the 2-D temperature distribution, conserves flux absolutely, and accurately computes scattering and polarization using arbitrary scattering phase functions. The output radiation field is binned into 10 polar intervals, i.e. each model produces 10 SEDs corresponding to different inclination viewing angles.

For each of a range of stellar masses, we define an evolutionary sequence of YSO models from what is traditionally called Class 0 (infalling flattened envelope with a narrow or no bipolar outflow cavity) to Class III (photosphere with a remnant debris disk). The sequence is characterized by decreasing envelope infall rate, increasing disk radius, increasing bipolar cavity opening angle, and decreasing cavity density. For infalling envelopes, we use the density prescription of a rotating envelope in free-fall collapse (eq.[1] of Whitney et al. 2003a). The disk is a flared disk in hydrostatic equilibrium. We note that we solve for the 3-D temperature in any specified circumstellar geometry; therefore we naturally compute a hot surface on the inner disk wall. In addition, our cavity dust is hot and has a high emissivity, despite its low density. Therefore our Class 0-II models include substantial mid-IR thermal emission from the warm disk and cavity regions.

Observational understanding of the dust distribution around young high-mass protostars is not very detailed, but it is not unreasonable to assume that there is rotationally flattened infall, and a dense structure that may resemble on average a disk. Thus, we scale our evolutionary sequence with the stellar mass to best match observations of massive protostellar objects with evidence of disks or rotating torii (Beltrán et al. 2004, Beuther et al. 2004, Sandell \& Sievers 2004, Sandell 2000, Shepherd, Claussen, \& Kurtz 2001). Further models are computed with large inner holes in the disk (relative to the dust descruction radius), especially for the higher-mass central objects. Some observations of protostellar disks suggest large inner holes, and planets can destroy the inner parts of their disks. The range of parameters present in the model grid is shown in Table 1. Example parameters for "Class I" objects are those used in Whitney et al. (2004) and noted here in Table 2; no preference is given to those parameters in fitting the data, but the values are reproduced here to give the reader an idea of the magnitudes of typical parameters, around which the model grid varies by at least an order of magnitude in all directions of circumstellar dust distribution parameter space.

We use similar grain properties as in Whitney et al. (2003b) (Table 3): a large-grain model for the high-density regions in the disk (Wood et al. 2002); a medium-sized grain model which fit the upper (scattering) layers of the HH30 disk (Cotera et al. 2001); and for the envelope and cavity, a grain model that gives an extinction curve typical of molecular clouds with $R_{V}$, the ratio of total-to-selective extinction, equal to 4.3. The dust sublimation temperature is chosen to be $1600 \mathrm{~K}$. We do not currently include emission 
Table 2. Example Parameters for Class I Models

\begin{tabular}{lllll}
\hline Stellar Temperature & 4000 & 8000 & 15000 & 30000 \\
Envelope infall rate $\left(/ 10^{-5} M_{\odot} / \mathrm{yr}\right)$ & 0.67 & 3.8 & 4.8 & 22 \\
Envelope mass $\left(M_{\odot}\right)$ & 0.12 & 8.5 & 31 & 1430 \\
Stellar radius $\left(R_{\odot}\right)$ & 4 & 11.6 & 5 & 7.3 \\
Stellar luminosity $\left(L_{\odot}\right)$ & 3.67 & 494 & 1134 & 46900 \\
Stellar mass $\left(M_{\odot}\right)$ & 0.5 & 6.0 & 6.2 & 20 \\
Envelope \& Disk inner radius $\left(R_{\star}\right)$ & 6.7 & 29 & 106 & 500 \\
Envelope \& Disk inner radius $(\mathrm{AU})$ & 0.125 & 1.55 & 2.47 & 16.9 \\
Envelope outer radius $(\mathrm{AU})$ & 3000 & 36900 & 59000 & 404000 \\
Disk mass $\left(M_{\odot}\right)$ & 0.01 & 0.30 & 0.31 & 1 \\
Disk outer radius $(\mathrm{AU})$ & 300 & 400 & 500 & 500 \\
Disk accretion rate $\left(/ 10^{-8} M_{\odot} / \mathrm{yr}\right)$ & 0.19 & 12.9 & 9.0 & 57 \\
Disk accretion luminosity $\left(/ 10^{-4} L_{a c c} / L_{\star}\right)$ & 1.5 & 0.73 & 0.15 & 0.01 \\
Cavity opening angle $(\mathrm{deg})$ & 20 & 20 & 20 & 20 \\
Cavity density $\left(/ 10^{-20} \mathrm{gm} \mathrm{cm}^{-3}\right)$ & 37 & 3.0 & 1.9 & 0.28 \\
$\tau_{V}(i=0$ deg) & 5 & 5 & 5 & 5 \\
$\tau_{V}(i=60 \mathrm{deg})$ & 25 & 25 & 25 & 25 \\
$\tau_{V}\left(i=90 \mathrm{deg}, / 10^{5}\right)$ & 1.93 & 2.43 & 1.26 & 0.40 \\
\hline
\end{tabular}

from polycyclic aromatic hydrocarbons (PAHs) or transiently heated grains; these likely affect fluxes in the IRAC wavelength range $(3-8 \mu \mathrm{m})$. The only source of calculation error in our models is photon counting statistics - running more photons produces higher signal-to-noise spectra. The models produced for figures in this paper took 12-24 hours each to run (on a $2 \mathrm{GHz} \mathrm{PC}$ and code compiled with g77). Draft quality results sufficient to explore trends take $\lesssim 2$ hours.

Each of thousands of point sources in a star forming region is compared to the grid of models, and a best-fit model is output, along with a best-fit foreground extinction (using the wavelength dependence of extinction measured from GLIMPSE data). Beyond the most-likely source classification, the distribution of models which fit reasonably well $\left(\chi^{2}\right.$ less than some threshold, e.g. 1) can be examined, to determine the uniqueness or reliability of the fit. This is illustrated in Whitney (this volume).

\section{Different Stages of star formation}

The GLIMPSE survey is rich with information on many stages of massive star formation, from prestellar cores through feedback and plausibly triggered second-generation star formation.

\subsection{IR-dark clouds: precluster clouds?}

Infrared dark clouds (IRDCs) consist of sizable collections of material on the near side of the Galaxy, opaque at $8-10 \mu \mathrm{m}$ and seen in silhouette against the Galactic disk. They were highlighted by the MSX mid-IR Galactic Plane survey (Egan et al. 1998, Carey et al. 2000), although had been seen by IRAS and ISOGAL (Hennebelle et al. 2001, Bacmann et al. 2000). The masses $\left(10^{4}-10^{5} \mathrm{M}_{\odot}\right.$, e.g. Carey et al. 1998) are consistent with being pre-cluster clouds, the high-mass equivalent of preprotostellar cores, and many of them show large lower-density envelopes in near-IR extinction, CO, and HI. A full understanding of how these objects fit in the evolution of massive stars is only now getting under way, with current directed surveys (e.g. Klein et al. and Won et al., this volume), and studies to measure physical conditions with molecular spectroscopy (e.g. $\mathrm{T}_{\text {rot }}\left(\mathrm{NH}_{3}\right) \simeq 10-20 \mathrm{~K}$, Pillai, this conference, and Chandler et al., in preparation). 

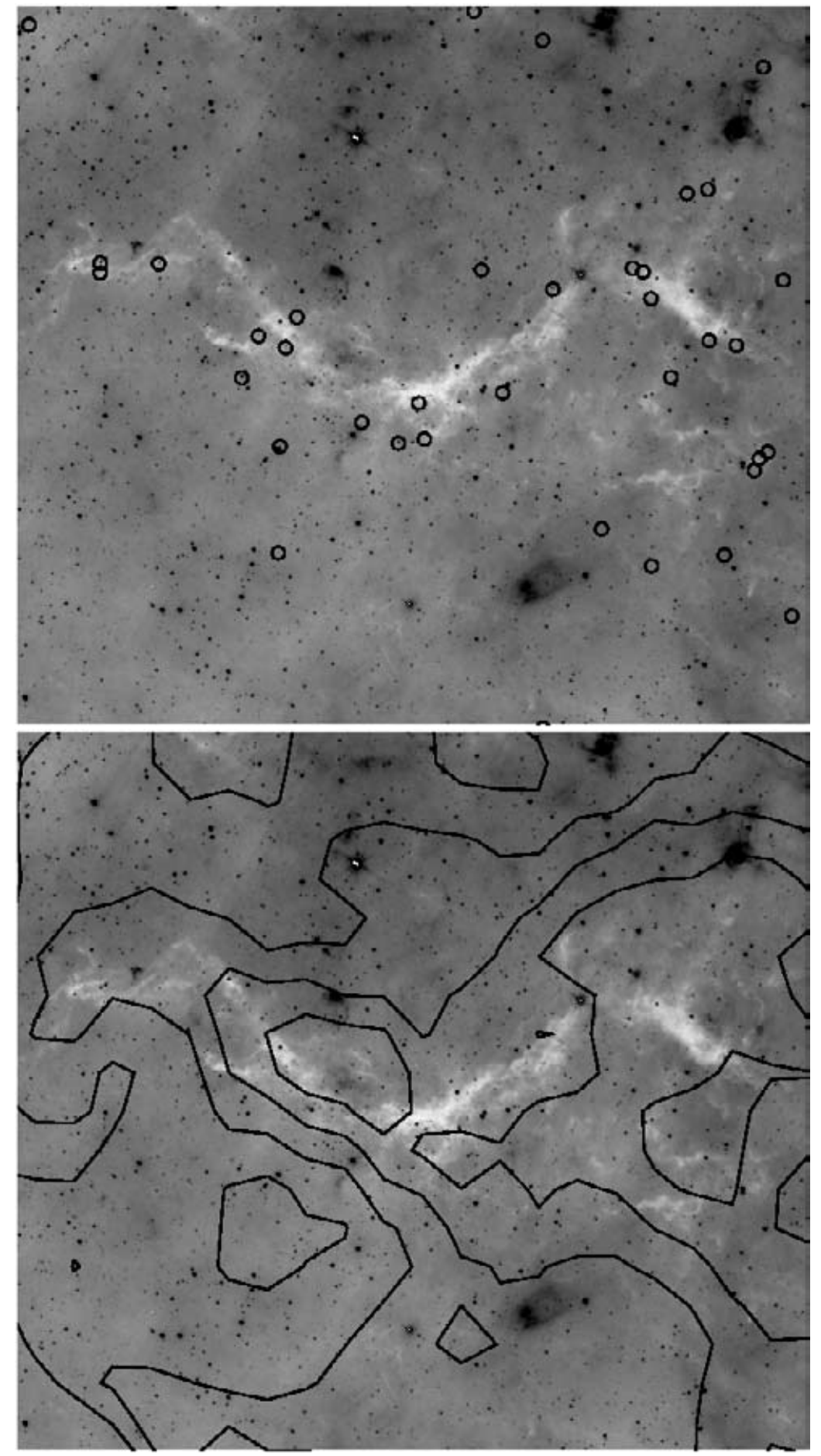

Figure 2. Infrared dark cloud near $l \simeq 11 \mathrm{deg}$. Point sources consistent with being protostars are marked in the first panel - there are not many, supporting the view of these clouds as young and mostly prestellar. The second panel shows contours of the extinction derived from the fitter the contour values are dominated by fitted extinction to photospheres, not YSOs. 
GLIMPSE can comment most easily on two aspects of IRDCs - the distribution and nature of associated protostars, and the distribution of column density, measured through extinction. Figure 2 shows a well-studied IRDC at $l \simeq 11 \mathrm{deg}$, with the locations of point sources whose SEDs are more consistent with protostars than with extincted photospheres (any evolutionary state from Class 0 through Class II, and also including IR-excess sources consistent with remnant disks). As mentioned above, the fitter also reports a value of the foreground extinction for all sources, and this can be used to map the extinction associated with a cloud or cluster, assuming that most of the extinction is associated with the cloud, or at least that foreground extinction along the line-of-sight does not change rapidly across the mapped region of the cloud. This method of producing an extinction map is similar to the NICE and NICER methods (Lada et al. 1994, Lombardi \& Alves 2001, Alves, this volume) which use near-IR color excess, but the use of GLIMPSE data at longer wavelengths should allow one to probe more deeply extincted regions.

\subsection{Star-forming Regions}

As motivated above, it is important to study the entire (proto)stellar population of starforming regions, and look for any correlations between spatial location, age, and stellar mass. Figures 3 and 4 show the galactic star-forming regions M16 and G305, with the locations of protostars and contours of extinction calculated from all of the sources in the field. Clear clusters of young objects are seen in M16, particularly in some of the pillars or elephant trunks. The space-mass-age distributions of the objects in these regions will tell us a great deal about the relationships between higher- and lower-mass star formation.

\subsection{Triggered star formation in $G 305$ and $R C W 79$ ?}

An important part of understanding massive star formation is determining the cause of molecular cloud collapse - spontaneous, or prompted/ triggered by nearby star formation or other source of compression. Figure 4 shows a giant HII region which has the ring-like infrared morphology suggestive of triggered star formation. A massive optically visible cluster resides near the middle of the ring, and clearly embedded objects are present on the edge. To quantify the effects, we calculate the overdensity of young stellar objects throughout the region - the space density of YSOs divided by the space density of main sequence stars. This highlights the areas in which there are relatively more protostars, rather than the uncorrected protostellar density, which tends to show the location of older clusters which happen to still have some associated star-formation activity. It also corrects for areas with high diffuse background, in which the effective sensitivity limit is lower, and fewer point sources of any type are detected. We see that there is an excess of star formation activity in the edges of the ring-like structure, although there is certainly star-formation activity elsewhere. A similar distribution is seen in RCW79, another ringlike region for which evidence of triggered star formation is presented by Zavagno, this volume.

\section{Conclusion}

We are entering an exciting new phase of infrared studies of nearby star formation, with publicly available surveys of the Galaxy (GLIMPSE and upcoming projects: GLIMPSE II IRAC survey of the inner Galaxy $(|l|<10 \mathrm{deg})$, SAGE LMC survey with MIPS and IRAC, and a MIPS survey of the GLIMPSE region). Tools such as an automated point source fitter and sophisticated radiative transfer models will allow this data 

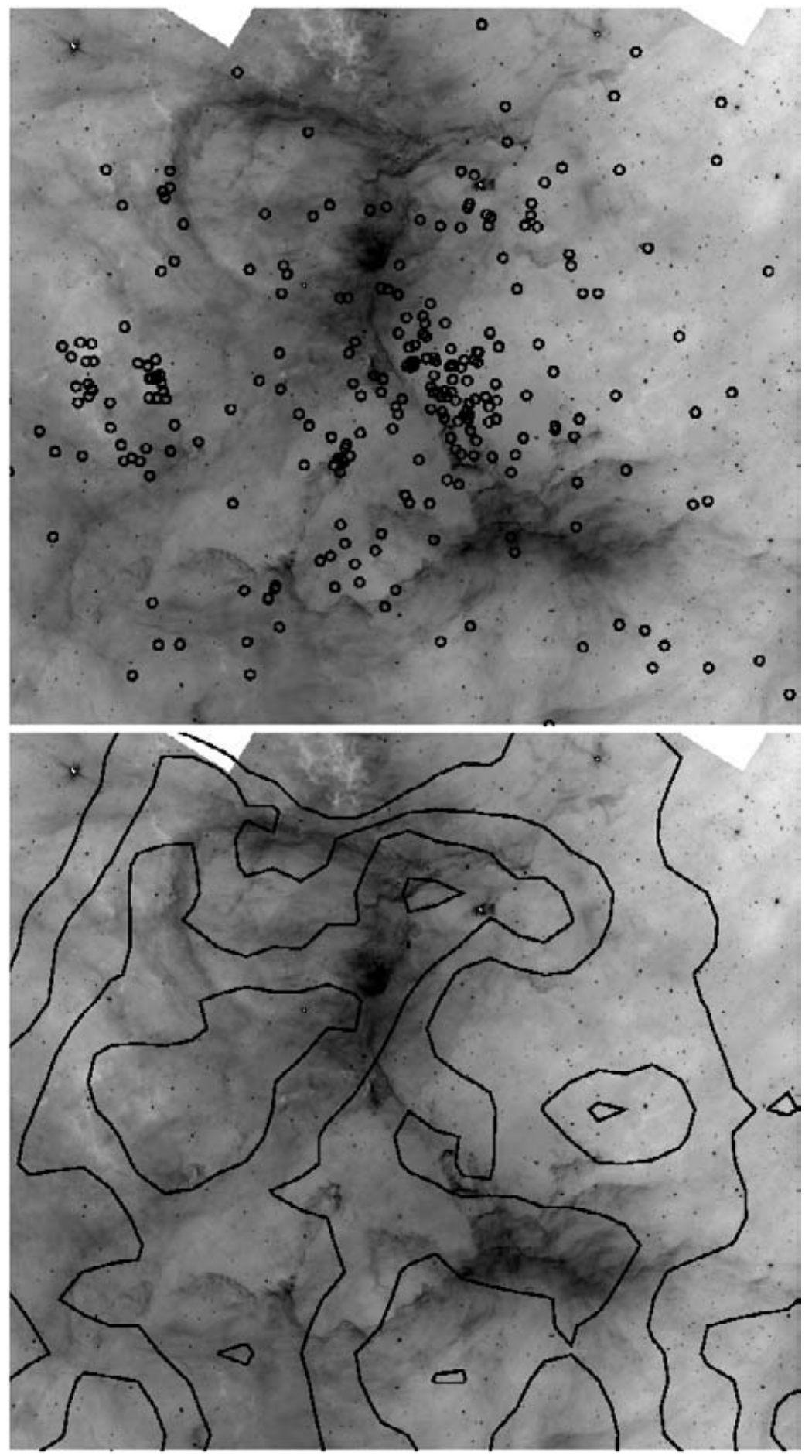

Figure 3. M16, as Figure 2. Protostars are marked in the first panel, and contours of extinction in the second panel. 


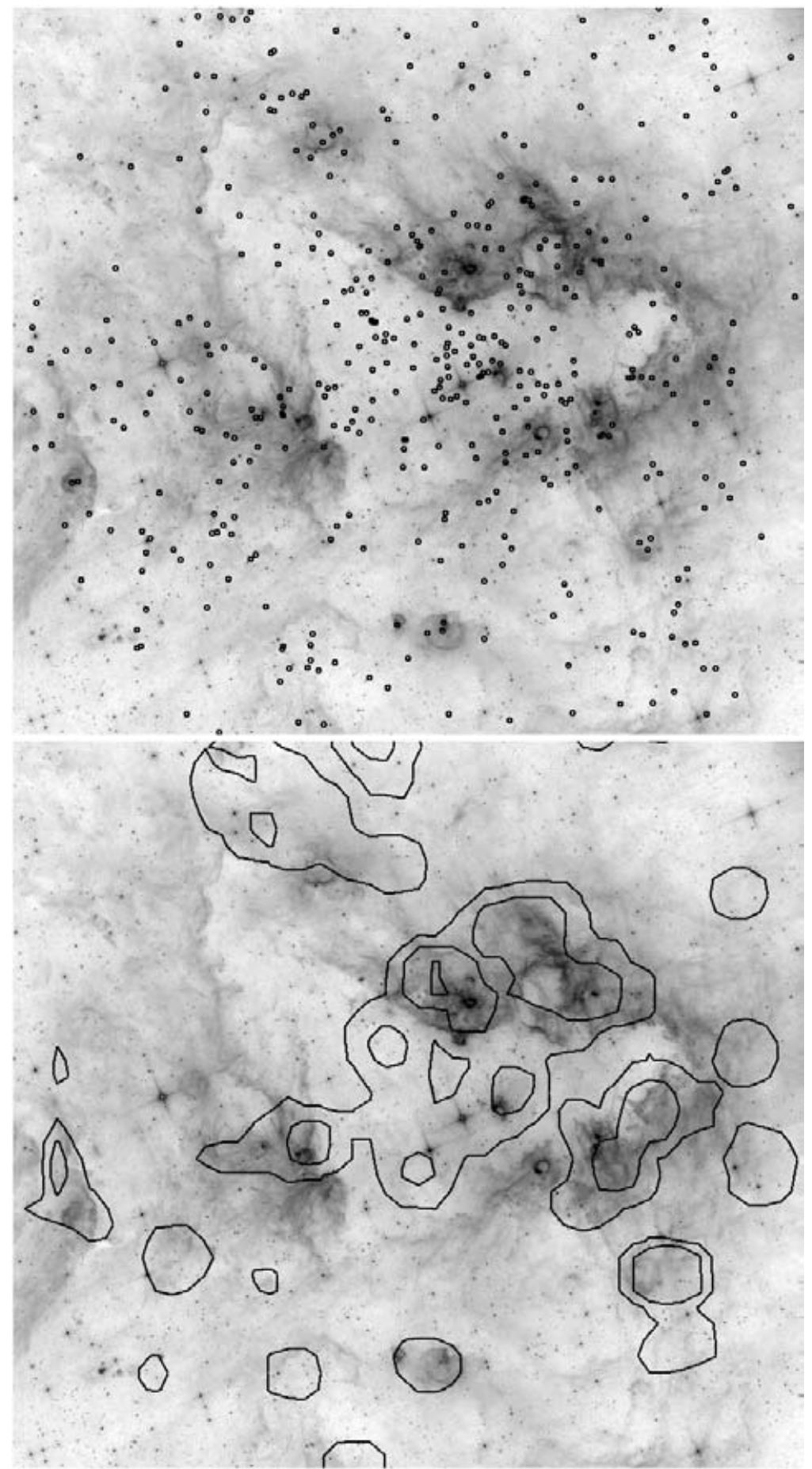

Figure 4. The first panel shows G305 with protostars marked as in the previous two figures. The second panel shows contours of protostellar excess - i.e. the density of protostars divided by the density of main-sequence stars in the region. 
to be exploited effectively to understand star formation in massive clusters and across the Galaxy and LMC.

\section{Acknowledgements}

The GLIMPSE project would not have been possible without the leardership of Ed Churchwell and the hard work of the data processing team based at the University of Wisconsin. RI is funded by a Spitzer Fellowship, and BW by NASA LTSA (NAG5-8933). We thank Ed Churchwell, John Mathis, and Jon Bjorkman for useful discussions on massive star formation, interstellar clumps, and radiative transfer.

\section{References}

Bacmann, A., André, P., Puget, J.-L., Abergel, A., Bontemps, S., \& Ward-Thompson, D. 2000, A\&Ap, 361, 555

Beltrán, M. T., Cesaroni, R., Neri, R., Codella, C., Furuya, R. S., Testi, L., \& Olmi, L. 2004, ApJL, 601, L187

Benjamin, R. A., et al. 2003, PASP, 115, 953

Beuther, H., et al. 2004, ArXiv Astrophysics e-prints, astro-ph/0402505

Bjorkman, J. E., \& Wood, K. 2001, ApJ, 554, 615

Cardelli, J. A., Clayton, G. C., \& Mathis, J. S. 1989, ApJ, 345, 245

Carey, S. J., Clark, F. O., Egan, M. P., Price, S. D., Shipman, R. F., \& Kuchar, T. A. 1998, ApJ, 508, 721

Carey, S. J., Feldman, P. A., Redman, R. O., Egan, M. P., MacLeod, J. M., \& Price, S. D. 2000, ApJL, 543, L157

Cotera, A. S., et al. 2001, ApJ, 556, 958

Draine, B. T. 2003, ARA\&A, 41, 241

Egan, M. P., Shipman, R. F., Price, S. D., Carey, S. J., Clark, F. O., \& Cohen, M. 1998, ApJL, 494, L199

Fazio, G., et al. 2004, ApJS,

Fitzpatrick, E. L. 1999, PASP, 111, 63

Hennebelle, P., Pérault, M., Teyssier, D., \& Ganesh, S. 2001, A\&Ap, 365, 598

Indebetouw, R., et al. 2005, ApJ, 619, 931

Jiang, B. W., Omont, A., Ganesh, S., Simon, G., \& Schuller, F. 2003, A\&Ap, 400, 903

Lada, C. J., Lada, E. A., Clemens, D. P., \& Bally, J. 1994, ApJ, 429, 694

Lombardi, M., \& Alves, J. 2001, A\&Ap, 377, 1023

Lutz, D., et al. 1996, A\&Ap, 315, L269

Maeder, A., \& Behrend, R. 2002, Ap\&SS, 281, 75

Motte, F., Andre, P., \& Neri, R. 1998, A\&Ap, 336, 150

Sandell, G. 2000, A\&Ap, 358, 242

Sandell, G., \& Sievers, A. 2004, ApJ, 600, 269

Shepherd, D. S., Claussen, M. J., \& Kurtz, S. E. 2001, Science, 292, 1513

Siess, L., Dufour, E., \& Forestini, M. 2000, A\&Ap, 358, 593

Tan, J. C., \& McKee, C. F. 2004, ApJ, 603, 383

Weingartner, J. C., \& Draine, B. T. 2001, ApJ, 548, 296

Whitney, B. A., Wood, K., Bjorkman, J. E., \& Wolff, M. J. 2003a, ApJ, 591, 1049

Whitney, B. A., Wood, K., Bjorkman, J. E., \& Cohen, M. 2003b, ApJ, 598, 1099

Whitney, B. A., Indebetouw, R., Bjorkman, J. E., \& Wood, K. 2004, ApJ, 617, 1177

Wood, K., Lada, C. J., Bjorkman, J. E., Kenyon, S. J., Whitney, B., \& Wolff, M. J. 2002, ApJ, 567,1183 